\title{
Flow autocorrelation: a dyadic approach
}

\author{
F. Bavaud . M. Kordi . C. Kaiser
}

Received: 31 October 2014 / Accepted: 16 January 2018

\begin{abstract}
The paper proposes and investigates a new index of flow autocorrelation, based upon a generalization of Moran's I, and made of two ingredients. The first one consists of a family of spatial weights matrix, the exchange matrix, possessing a freely adjustable parameter interpretable as the age of the network, and controlling for the distance decay range. The second one is a matrix of chi-square dissimilarities between outgoing or incoming flows. Flows have to be adjusted, that is their diagonal part must first be calibrated from their off-diagonal part, thanks to a new iterative procedure procedure aimed at making flows as independent as possible. Commuter flows in Western Switzerland as well as migration flows in Western US illustrate the statistical testing of flow autocorrelation, as well as the computation, mapping and interpretation of local indicators of flow autocorrelation. We prove the present dyadic formalism to be equivalent to the "origin-based" tetradic formalism found in alternative studies of flow autocorrelation.
\end{abstract}

\section{JEL classification codes: C21, C23, R12}

Keywods: diffusive spatial weights, exchange matrix, flow autocorrelation, movers-stayers, weighted network

\section{Introduction}

Flows $n_{i k}$ count the number of units (people, goods, information, money etc.) at origin $i$ and destination $k$ after some time $t$. Flows are autocorrelated if the (suitably normalized) counts of a pair of spatially close flows tend to be more similar than the counts of a pair of flows chosen at random. Assessing

Institute of Geography and Sustainability, University of Lausanne, Switzerland E-mail: francois.bavaud@unil.ch, maryam.kordi@gmail.com, christian.kaiser@unil.ch 
the spatial proximity between two flows requires in general the construction of tetradic neighborhood operators (adjacencies, spatial weights or exchange matrices), involving two origins and two destinations (section 4).

By contrast, most studies on spatial autocorrelation compare the features of a region to the features of a spatially close region, and involve only dyadic neighborhood operators. Spatial autocorrelation is most often measured by Moran's I. A generalization of the latter, particularly useful for multivariate features and weighted networks, is provided by the relative autocorrelation $\delta$

$$
\delta:=\frac{\Delta-\Delta_{\mathrm{loc}}}{\Delta} \quad \Delta:=\frac{1}{2} \sum_{i, j=1}^{n} f_{i} f_{j} D_{i j} \quad \Delta_{\mathrm{loc}}:=\frac{1}{2} \sum_{i, j=1}^{n} e_{i j} D_{i j}
$$

where $\Delta$ and $\Delta_{\text {loc }}$ respectively constitute the (weighted) global and local inertia. Here $D_{i j}$ is a squared Euclidean dissimilarity between the attributes or features of regions $i$ and $j$, and $E=\left(e_{i j}\right)$, the exchange matrix, is the symmetric, normalized spatial weights matrix (e.g. Bavaud 2013), whose margin $f_{i}$ yields the relative weight of region $i$ (section 2.1).

This paper proposes an original procedure aimed to measure and test flow autocorrelation, based upon dyadic formalism (1). The latter is demonstrated to be equivalent to the "origin-based" tetradic formalism, that is based upon spatial and attributes comparison operators $e_{i j, k l}$ and $D_{i j, k l}$, discussed in prevailing studies of flow autocorrelation (section 4). Technical as it might appear, the procedure however relies upon two $n \times n$ data sets only, namely a proximity or generalized adjacency matrix, and a flow matrix, whose diagonal parts are irrelevant in both cases (section 2).

The approach is illustrated on a commuting flow dataset for Western Switzerland, as well as a migration dataset for Western US (section 3). Both examples exhibit highly significative autocorrelation. Local indicators of flow autocorrelation (Anselin 1995) can also be defined (section 2.3), mapped and interpreted in terms of properties of origins and destinations. For commuters flow, they highlight in particular the role of residential zones versus job centers, within a given, freely adjustable spatial resolution.

\subsection{A descriptive perspective}

Measuring flow autocorrelation is not that trivial, and arguably justifies contributions such as the present one, exclusively devoted to the formal definition and measure of the relative flow autocorrelation, free of socio-economic variables aimed at explaining this state of affairs: specifying measurement should logically improve model selection - hence the current emphasis on the computational procedure. Section 4 contains references adopting a more econometric perspective, and section 3 resorts to geographical interpretation of the local index of flow autocorrelation defined in section 2.3. 
In a nutshell, the proposed measure of flow autocorrelation is constructed as follows: $D_{i j}$ in (1) is chosen as the chi-square dissimilarity between out-going (normalized) flows with origins $i$ and $j$, or alternatively between in-coming flows. Also, $e_{i j}$ in (1) measures the spatial proximity between regions $i$ and $j$. Two auxiliary problems, interesting in themselves, have yet to be further solved at this stage:

- diagonal flows $n_{i i}$, counting stayers, are not always recorded, and their contribution to flow autocorrelation is debatable anyway. Section (6) proposes an original iterative calibration procedure of diagonal flows from off-diagonal flows, counting movers, aimed at computing sensible flow dissimilarities between origins (or destinations).

- the spatial structure is generally given in terms of proximities or adjacencies, and the exchange matrix $\left(e_{i j}\right)$, whose margins must coincide with the given regional weights $\left(f_{i}\right)$, has still to be constructed accordingly. Section (2.1) gives a general recipe, producing diffusive exchange matrices generated by jump processes characterized by "generalized adjacency matrices" (Bavaud 2014), specifying which weighted regions are immediately attainable from which ones. Iterating the process permits to attain any region in the long run, and to assess the probability to do so.

In summary, measuring and testing flow autocorrelation implies the following steps:

1) after adjusting for the diagonal flows (sections 2.4 and 6), compute the origin weights $f$, the destination weights $\rho$, and the chi2 flow dissimilarity $D$ between pairs of origins - or destinations (section 2.2)

2) compute the weight-compatible exchange matrix $E(f, G, t)$ from a proximity matrix $G$, such as the adjacency matrix (section 2.1). Here $t$ is a freely adjustable diffusion time, ranging from $t=0$ (absence of exchange, complete autarchy) to $t=\infty$ (absence of spatial friction, complete mobility)

3 ) compute and test relative autocorrelation $\delta(E, D)$, using normal approximation or a permutation approach (section 2.3)

4) compute and map local indices of flow autocorrelation $\delta_{i}(E, D)$ (section 3 ).

\section{Notations and formalism}

Recall that $e_{i j}$ in (1) expresses spatial neighborhoodness between a pair of origin regions $i$ and $j$, and $D_{i j}$ expresses their dissimilarities, comparing their normalized, off-diagonal destination flow profiles.

2.1 The exchange matrix $E$ and its generation from a proximity matrix $G$

As already stated, a weighted, unoriented network of $n$ regions is defined by an $n \times n$ symmetric, normalized, weight-compatible exchange or spatial weights 
matrix $E=\left(e_{i j}\right)$, inducing compatible regional weights $f_{i}$ as

$$
e_{i j}=e_{j i} \geq 0 \quad \sum_{j=1}^{n} e_{i j}=e_{i \bullet}=f_{i} \quad e_{\bullet \bullet}=\sum_{i=1}^{n} f_{i}=1
$$

(here and in the sequel, "•" denotes the summation over the values of the replaced index). The exchange matrix $E=\left(e_{i j}\right)$ is a normalized measure of spatial interaction, interpretable as the probability to select the pair of regions $(i, j)$ (Berger and Snell 1957). E defines an unoriented weighted network, whose (compatible) node weights $f_{i}$ obtain as the sum of the incident edge weights.

Let $\Pi=\operatorname{diag}(f)$ denote the diagonal matrix of regional weights, and consider a proximity $n \times n$ symmetric matrix $G$, essentially non-negative (that is with $g_{i j} \geq 0$ for $i \neq j$ ) expressing spatial proximity between regions. In both case studies (section 3), $G$ is simply taken as the binary adjacency matrix between regions.

As a continuous-time generator, $G$ defines a diffusive, weight-compatible exchange matrix $E$ by means of the matrix exponential

$$
E(G, f, t):=\Pi^{1 / 2} \exp (-t \Psi) \Pi^{1 / 2} \quad \text { with } \quad \Psi:=\Pi^{-1 / 2} \frac{L G}{\operatorname{trace}(L G)} \Pi^{-1 / 2}
$$

where $L G$ is the Laplacian of $G$ (e.g. Chung 1997 p.12), with components $(L G)_{i j}:=\delta_{i j} g_{i \bullet}-g_{i j}$ (Kronecker's delta $\delta_{i j}$ denotes the components of the identity matrix). By construction, the resulting exchange matrix, whose computation can be achieved by spectral decomposition, is non-negative and weightcompatible (Bavaud 2014). It turns out to be positive semi-definite as well. The free parameter $t>0$ interprets as the age of the network, and controls the importance of diagonal weights: indeed, $\operatorname{trace}(E(G, f, t))=1-t+O\left(t^{2}\right)$. One gets $\lim _{t \rightarrow 0} e_{i j}(G, f, t)=f_{i} \delta_{i j}$ ("frozen network" made of $n$ non-interacting regions) and $\lim _{t \rightarrow \infty} e_{i j}(G, f, t)=f_{i} f_{j}$ ("complete network", free of distancedeterrence effects). Construction (2) constitutes a weight-compatible extension of the so-called diffusion kernel of machine learning (Kondor and Lafferty 2002) from general proximity matrices.

\subsection{Specifying regional differences by squared Euclidean dissimilarities}

Consider also a $n \times n$ matrix of squared Euclidean dissimilarities $D=\left(D_{i j}\right)$ between the regional characteristics, features or attributes, that is of the form

$$
D_{i j}=\left\|x_{i}-x_{j}\right\|^{2} .
$$

As a simple example, contemplate $D_{i j}=\left(x_{i}-x_{j}\right)^{2}$ where $x$ is a density variable, that is transforming as a weighted average under regional aggregation $i, j \rightarrow[i \cup j]$, as with $x=$ "average life span", "proportion of retired people" or "average housing surface per individual". 
Taking simultaneously into account many numerical variables yields to multivariate dissimilarities of the form $D_{i j}=\sum_{k}\left(x_{i k}-x_{j k}\right)^{2}=\left\|x_{i}-x_{j}\right\|^{2}$, where $x_{i k}$ is a standardized density score of region $i$ on feature $k$.

In the presence of a categorical variable with $p$ modalities indexed by $k$, the matrix $N=\left(n_{i k}\right)$, counting the number of units of region $i$ possessing the modality $k$, can generate various kinds of squared Euclidean dissimilarities, most notably the normalized chi-square dissimilarity

$$
D_{i j}^{\chi}=\sum_{k=1}^{p} \frac{n_{\bullet \bullet}}{n_{\bullet k}}\left(\frac{n_{i k}}{n_{i \bullet}}-\frac{n_{j k}}{n_{j \bullet}}\right)^{2} \quad \text { obeying } \quad \frac{1}{2} \sum_{i j} \frac{n_{i \bullet}}{n_{\bullet \bullet}} \frac{n_{j \bullet}}{n_{\bullet \bullet}} D_{i j}^{\chi}=\frac{\text { chi2 }}{n_{\bullet \bullet}}=\Delta
$$

Commuter flows $T=\left(t_{i k}\right)$ could seemingly be directly plugged into (3) in place of $N=\left(n_{i k}\right)$, in order to obtain chi-square dissimilarities between origins $i$ and $j$. However, the particular nature of diagonal flows $t_{i i}$ (section 1.1) calls for a prior, somewhat stable recalibration of diagonal counts in $T$ (stayers) from the off-diagonal counts (movers) only (section 2.4 and appendix 6).

\subsection{Measuring and testing spatial autocorrelation}

The relative autocorrelation $\delta$ in (1) constitutes a weighted, multivariate generalization of Moran's $I$. The global inertia $\Delta$ and the local inertia $\Delta_{\text {loc }}$ obey $0 \leq \Delta_{\text {loc }} \leq 2 \Delta$, thus making $-1 \leq \delta \leq 1$. Also, the relative autocorrelation can also be expressed as the average of a local indicator of spatial association $\delta_{i}$ (Anselin 1995). In the present formalism ${ }^{1}$ :

$$
\delta_{i}:=\frac{1}{2 \Delta} \sum_{j}\left(f_{j}-w_{i j}\right) D_{i j} \quad w_{i j}:=\frac{e_{i j}}{f_{i}}=P(j \mid i) \quad \delta=\sum_{i} f_{i} \delta_{i}
$$

where $W=\left(w_{i j}\right)$ is the transition matrix of a reversible Markov chain, the rowstandardized spatial weights (e.g. Bavaud 1998; Bivand et al. 2008) appearing in spatial econometrical models such as $y=\rho W y+\beta X+\epsilon$. By construction, $\delta_{i}>0$ iff spatial transitions from $i$ preferentially reach neighboring regions $j$ whose attributes are more similar to $i$ than are regions chosen at random.

Under the null hypothesis $H_{0}$ of absence of spatial autocorrelation, the expected value of the autocorrelation index, together with its variance (under normal approximation) are (e.g. Cliff and Ord 1981; Bavaud 2013)

$$
E_{0}(\delta)=\frac{\operatorname{trace}(W)-1}{n-1} \quad \operatorname{Var}_{0}(\delta)=\frac{2}{n^{2}-1}\left[\operatorname{trace}\left(W^{2}\right)-1-\frac{(\operatorname{trace}(W)-1)^{2}}{n-1}\right]
$$

1 other possible choices such as $\delta_{i}=(W B)_{i i} / \Delta$, where $B$ is the matrix of scalar products associated to $D$, will be discussed in a forthcoming publication. 
where $W$ is the above Markov transition matrix. Hence the relative autocorrelation is significant at level $\alpha$ (two-tailed test) if

$$
|z| \geq u_{1-\alpha / 2} \quad \text { where } \quad z:=\frac{\delta-E_{0}(\delta)}{\sqrt{\operatorname{Var}_{0}(\delta)}},
$$

and $u_{\alpha}$ denotes the $\alpha$-th quantile of the standard normal distribution. Decision rule (6) is well adapted for a large number of weighted regions (Bavaud 2013). A meticulous assessment of the validity of normal approximation for multivariate dissimilarities is beyond the scope of the present study, and should here in particular address the issues of integer counts (in particular zero counts), as well as the impact of their (in-)dependence on the distribution of chi2 dissimilarities. Yet, the sheer size of the $z$ values encountered in both cases studies (section 3) demonstrate a posteriori the presence of massive, very significant flow autocorrelation.

\subsection{Diagonal flow calibration: estimating stayers from movers}

In any case, the proposed procedure consists in considering mover flows only, defined by $m_{i j}=t_{i j}$ for $i \neq j$, and $m_{i i}=0$, and in estimating the stayer flows $t_{i i}$ by requiring the complete flow $t_{i j}$ to be as origin-destination independent as possible. Specifically, define the normalised empirical distribution of movers as

$a_{i j}:=\frac{m_{i j}}{m_{\bullet \bullet}}=\frac{t_{i j}\left(1-\delta_{i j}\right)}{\operatorname{sum}(T)-\operatorname{trace}(T)} \quad$ (proportion of movers from $i$ to $\left.j \neq i\right)$

Assume the relative complete flow $P=\left(p_{i j}\right)$, normalised to $p_{\bullet \bullet}=1$, to be of the form

$$
p_{i j}:=\frac{t_{i j}}{t_{\bullet \bullet}}=\sigma_{i} \delta_{i j}+\mu a_{i j} \quad \text { where } \mu \geq 0, \sigma_{i} \geq 0 \text { and } \mu=1-\sigma_{\bullet} .
$$

In particular, $\operatorname{trace}(T) / \operatorname{sum}(T)=\sigma_{\bullet}=1-\mu=1-t-0\left(t^{2}\right)$, which makes $\mu$, when small, comparable to the age $t$ of a "diffusive" network (Bavaud 2104): $\mu=t=0$ generates pure stayer "flows" (absence of true commuters, "frozen" network). By contrast, $\mu=1$ characterizes complete bimodal segregation of regions into "exclusive origins" and "exclusive destinations" (migrations), or into "pure homeplaces" and "pure workplaces" (commuters), arising whenever $a_{i \bullet} a_{\bullet}=0$ for all regions $i=1, \ldots, n$ (see the appendix in section 6 ).

\subsubsection{Adjusted flows: regional weights and uncertainties}

The appendix presents a presumably original method of stayers calibration, determining the normalised adjusted relative flows $p_{i k}=\sigma_{i} \delta_{i k}+\mu a_{i k}$ by iteration. Once obtained, $p_{i k}$ can be plugged into (3) in place of the $n_{i k}$, finally enabling the computation of chi-square dissimilarities $D_{i j}^{\chi}$ between origins $i$ and $j$. 
Working with transposed flows $T^{\prime}$ instead yields the construction of chi-square dissimilarities between destinations. Origin weights obtain as $f_{i}:=p_{i \bullet}$, destination weights as $\rho_{k}:=p_{\bullet k}$. Uncertainties on origins $O$, on destinations $D$, and on origin-destination trips are measured (in nats) by the entropies

$H(O)=-\sum_{i} f_{i} \ln f_{i} \quad H(D)=-\sum_{k} \rho_{k} \ln \rho_{k} \quad H(O, D)=-\sum_{i k} p_{i k} \ln p_{i k}$

Mutual information $I(O: D):=H(O)+H(D)-H(O, D)$ constitutes another measure of origin-destination dependence, approximatively equal to $\Delta / 2$ for flows close to independence (e.g. Cover and Thomas 1991). Also, the "uncertainty unbalance"

$$
\Delta H:=H(O)-H(D)=H(O \mid D)-H(D \mid O)
$$

is positive iff the origin of people are more difficult to guess than their destination, i.e. iff origins are less concentrated than destinations.

\section{Case studies}

Commuters (Western Switzerland, section 3.1) and migrants (US Westcoast, section 3.2) turn out to be contrasted, in that $\Delta H>0$ for commuters flow, while $\Delta H<0$ for migratory flow. Commuters destinations happen to be more concentrated than commuters origins - an intuitively expected result. The opposite holds for migratory flow, where the question "where are you going" appears to be more challenging than "where are you from". Further studies could examine to which extent $\Delta H<0$ may characterize other migratory flows.

In both cases, recall that the exchange matrix $E(G, f, t)(2)$ between origins (that is for out-flows) has been constructed from the binary adjacency matrix $G$ between regions, where $f$ are the origin weights and the free parameter $t$ represents the "age of the network", determining the importance of the distance deterrence effect. Computations have been performed with $t=0.05$ and $t=1$, the latter representing a network where spatial weights become nearly independent on geographical distance. Time-reversal $T \rightarrow T^{\prime}$ similarly yields the exchange matrix $\dot{E}(\rho, G, t)$ between destinations, weight compatible in the sense $\dot{e}_{j \bullet}=\rho_{j}$.

Spatial autocorrelation is extremely significant for both flows, in either direction, that is for out- and in-flows. Local indicators $\delta_{i}$ of spatial autocorrelation appear fairly spatially continuous (especially for migratory flows), i.e. spatially autocorrelated in turn. Their two versions (out- and in-flows) exhibit similar patterns, high values of $\delta_{i}$ seemingly underlying regions important as origins, respectively as destinations. 


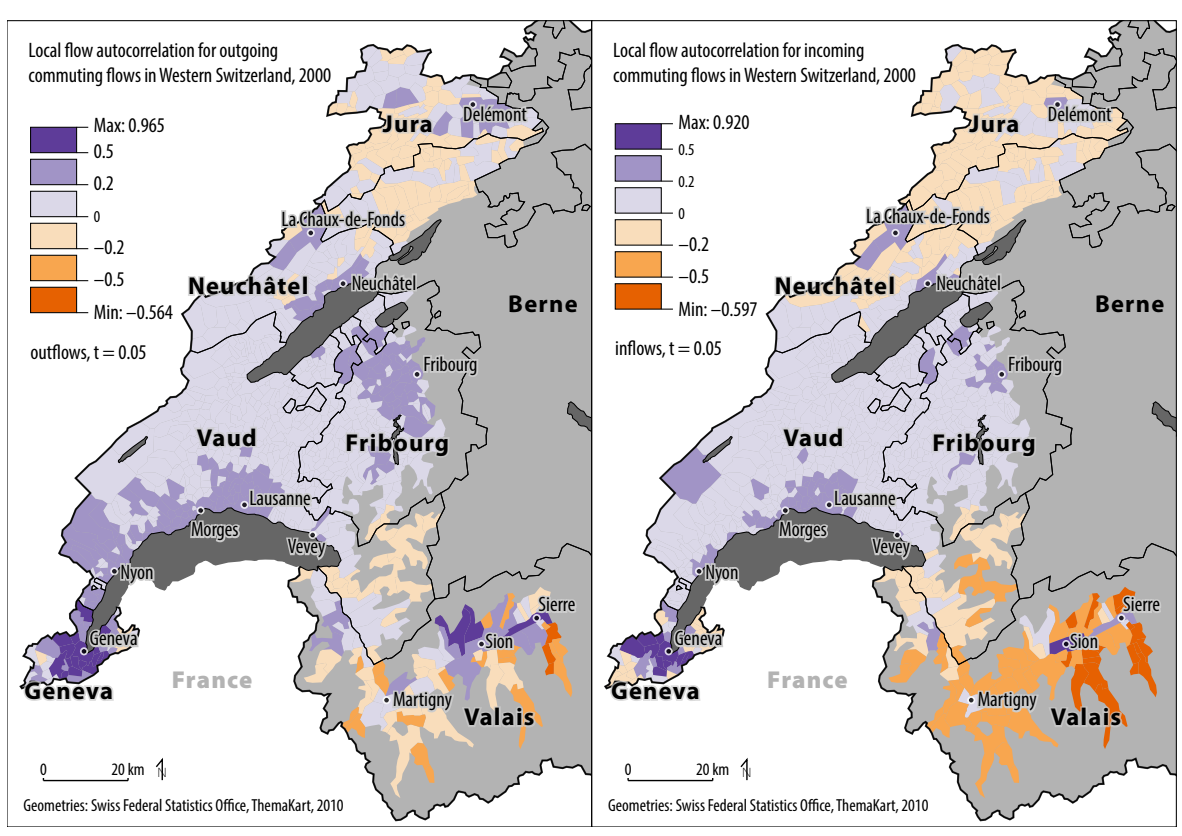

Fig. 1 Local indicator of flow autocorrelation $\delta_{i}(4)$ for commuting flows in Western Switzerland $(n=895)$, for $t=0.05$, outflows (left) and inflows (right).

\begin{tabular}{ccc} 
& $\mathbf{t}=\mathbf{0 . 0 5}$ & $\mathbf{t}=\mathbf{1 . 0}$ \\
\hline \hline outflows & $\delta=0.207$ & $\delta=0.024$ \\
& $z=80.93$ & $z=80.20$ \\
\hline inflows & $\delta=0.177$ & $\delta=0.025$ \\
& $z=69.07$ & $z=73.60$ \\
\hline
\end{tabular}

Table 1 Relative flow autocorrelation (commuting flows in Western Switzerland) for outflow and inflow studies, and standardised normal decision variable $z$ for testing $H_{0}$ : "flows are not spatially autocorrelated", with two different values for parameter $t$

\subsection{Commuting flows in Western Switzerland}

Journey-to-work flows for Western Switzerland (for the year 2000) are provided by the Swiss Federal Statistical Office $(\mathrm{SFSO})^{2}$. The dataset contains more than 38'000 non-zero flows between the $n=895$ French-speaking communes of Western Switzerland, communes being the smallest administrative division corresponding mostly to a town or a city. Removing the stayer flows yields a total of 37'378 non-zero mover flows, representing 733'164 people.

We have computed the relative flow autocorrelation $\delta$ on both outgoing and incoming flows, for two different values of $t$ used in deriving the exchange matrix $E$ based on the adjacency matrix $G$ (section 2.1).

2 Data available at http://www.pendlerstatistik.admin.ch 


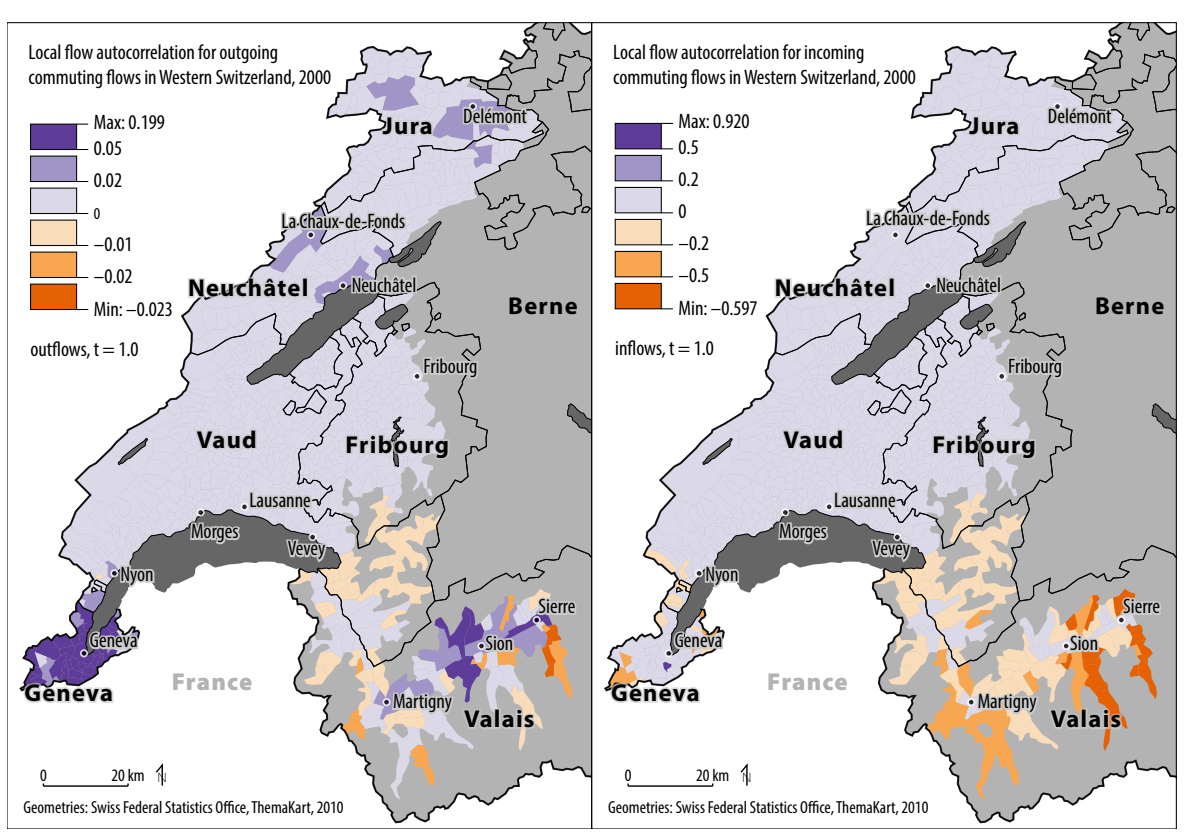

Fig. 2 Local indicator of flow autocorrelation $\delta_{i}$ for commuting flows in Western Switzerland, for $t=1.0$, outflows (left) and inflows (right).

Table 1 exhibits the relative flow autocorrelation $\delta$ for the different configurations; in all cases, the significance of (positive) flow autocorrelation is overwhelming $(p \simeq 0)$. Figure 1 depicts the local indicator of flow autocorrelation or outflows (left) and inflows (right) for a value of $t=0.05$, while figure 2 displays the same information but for a value of $t=1$.

Figure 1 shows well the economic centres of the region, mainly Geneva and Lausanne, and some local centres like Fribourg or Sion. Recall that, in the present setup, a large value of $\delta_{i}$ for outflows indicates that the profile of outflows emanating from neighbours $j$ of $i$ is similar to the profile of outflows from $i$ itself - a circumstance favorable to car-sharing. This is typically the case of the metropolitan area of Geneva. Remote areas exhibit a negative flow autocorrelation, typically remote Alpine valleys south of Sion or Sierre.

The same comments apply to inflows, modulo a reversal of origins and destinations. Outflow local autocorrelation primarily highlights residential zones where people are living, while inflow local autocorrelation $\delta_{i}$ is high for economically strong communes (job centres) and low for economically weak places. Hence, our local indicator for flow autocorrelation is clearly able to highlight the spatial hierarchical structure underlying the journey-to-work flows.

Figure 2 shows a much more equal spatial distribution for the local indicator of flow autocorrelation than figure 1: a large value of $t$ corresponds to moderate distance deterrence effect and large neighborhoods, filtering out local details of smaller size. Only the Alpine canton of Valais (with Martigny, 
Sion and Sierre) is well separated, as well as Geneva for the outflows. Both the case of Geneva and Valais are a bit special for transportation because they have only a single access route from outside, due to topography (Valais) or administrative boundaries (Geneva) with France being close.

As shown by table 1, spatial autocorrelation of inflows is huge, and spatial autocorrelation of outflows is even larger. This difference could be explained by the relatively larger difficulty to guess the origin of a commuter of Western Switzerland, compared to the guess of its destination, as measured by their entropies $H(O)=6.61$ nats, versus $H(D)=6.35$ nats. Equivalently, guessing the residential place of a commuter at its job place is more difficult than the other way round: $H(O \mid D)=5.98>H(D \mid O)=5.72$, thus making outflows somewhat more focused than inflows. The spatial distribution of jobs respectively residential zones might be at the source of this difference. Jobs are typically more concentrated in urban centres and in some limited suburban areas, while residential zones are distributed over a wider region, hence the bigger difficulty to guess an origin than a destination.

\subsection{County-to-county migration flows in Western United States}

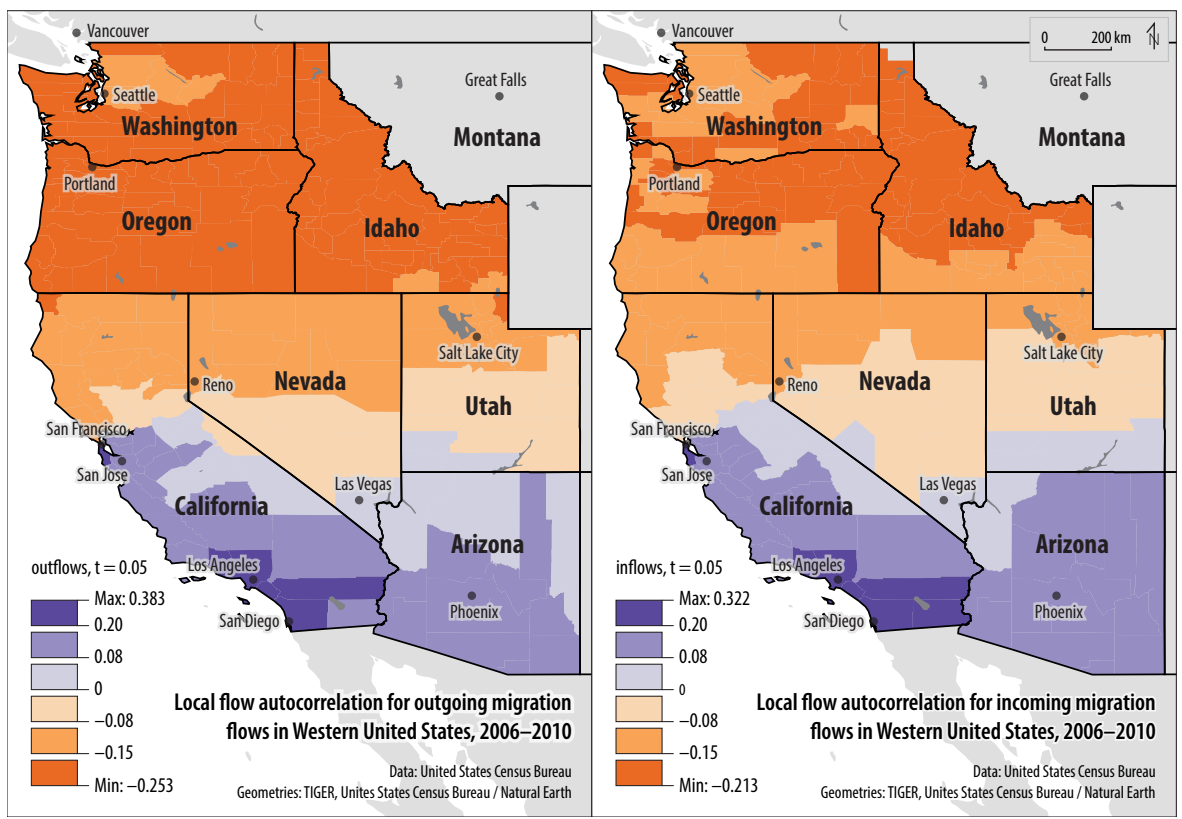

Fig. 3 Local indicator of flow autocorrelation $\delta_{i}$ for migration flows for the Western United States $(n=237)$, for $t=0.05$, outflows (left) and inflows (right). 


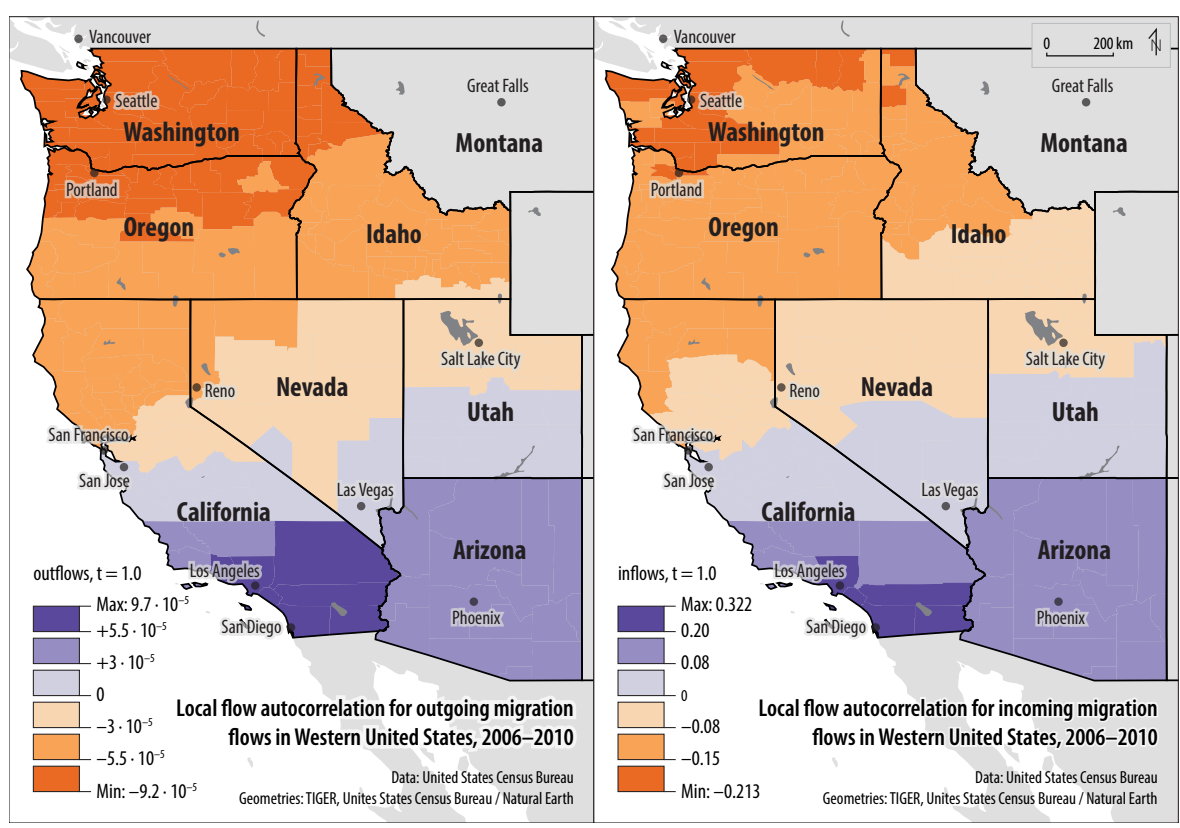

Fig. 4 Local indicator of flow autocorrelation $\delta_{i}$ for migration flows for the Western United States, for $t=1.0$, outflows (left) and inflows (right).

\begin{tabular}{ccc} 
& $\mathbf{t}=\mathbf{0 . 0 5}$ & $\mathbf{t}=\mathbf{1 . 0}$ \\
\hline \hline outflows & $\delta=0.080$ & $\delta=1.3 \cdot 10^{-5}$ \\
& $z=13.27$ & $z=9.18$ \\
\hline inflows & $\delta=0.076$ & $\delta=1.1 \cdot 10^{-5}$ \\
& $z=13.29$ & $z=9.03$ \\
\hline
\end{tabular}

Table 2 Relative flow autocorrelation (US counties migration flow westcoast) for outflow and inflow studies, and standardised normal decision variable $z$, with two different values for parameter $t$

County-to-county migration flow estimates are provided by the U.S. Census Bureau based on the 2006-2010 American Community Survey program ${ }^{3}$. While the original dataset contains flow estimates for all U.S. counties and Puerto Rico, in this case study, we use a subset covering the Western states of Washington, Oregon, Idaho, California, Nevada, Utah and Arizona. A total of $n=237$ counties are considered, resulting in nearly 10'000 non-zero flows and more than 2.2 million of migrants for a total population of a bit more than 61 million residents.

As stated above, origins are more concentrated than destinations for the Western US migration flows: $H(O)=4.23$ and $H(D)=4.36$. Also, $H(O \mid D)=$ 3.11 and $H(D \mid O)=3.24$. This might betray dominating push-effects over

3 Data available at http://www.census.gov/hhes/migration/data/acs/county_to_ county_mig_2006_to_2010.html 
pull-effects: typically, the key factor for migration may be the lack of job possibilities or educational infrastructure rather than the presence of it, in which case people primarily choose "just to leave a given origin" rather than "going to a specific destination". It also shows that the destination is more difficult to "guess", which might be related to the presence of many possible job centres attracting migrants. For journey-to-work flows, (time) distance between home and work is a key factor, while this does not necessarily hold for county-to-county migration flows. It is more likely that economic differences and possibly personal preferences or opportunities are the key drivers behind migration.

Except for this notable difference, most comments on Swiss commuters still hold for the present case. In particular, the spatial autocorrelation of both outand in-flows is strongly significant (table 2), although to a lesser extent than for commuter flows. The local autocorrelation is spatially continuous, and follows a systematic North-South gradient, highest for the more populated regions of South and especially for the South-West around Los Angeles. For the Northern parts of the study zone the local spatial autocorrelation is negative, more for outflows than inflows. Especially for inflows, local spatial autocorrelation is slightly less negative for the areas around Seattle and Portland. Densely populated areas tend to present a higher spatial autocorrelation than surrounding zones (figure 5). Some state boundaries are remarkably well respected, possibly due to administrative artifacts or to true inter-state migration barriers - a much debated issue in spatial econometrics (e.g. Hillberry and Hummels (2008); Llano-Verduras et al. (2011)).

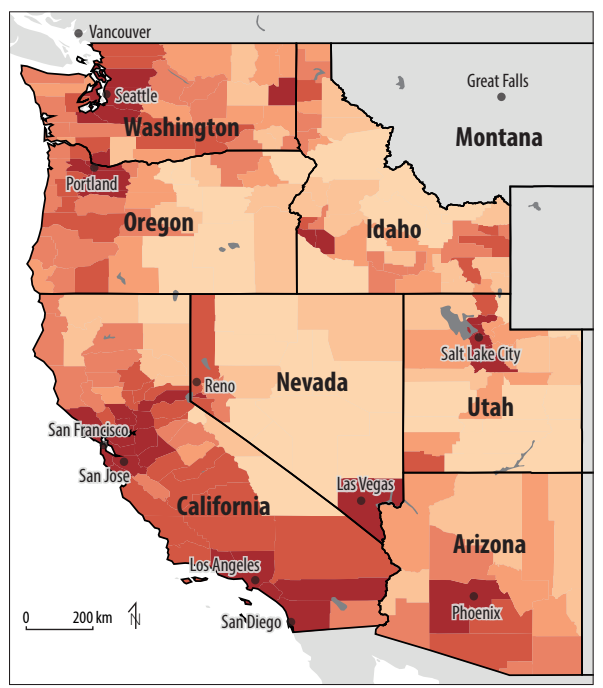

Population density in Western US, 2010

Population density in number of residents per square kilometer, per county

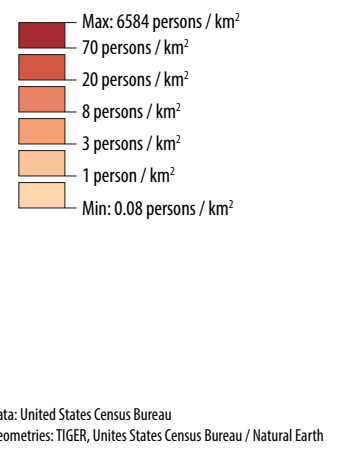

Fig. 5 Population density in 2010 for the counties of Western US. Population density can explain parts of the spatial autocorrelation, especially for inflows, due to the economic attractiveness of urban areas and their importance for migration. 


\section{Equivalence between the dyadic and tetradic approaches}

Spatial autocorrelation $\delta(x)$ of, say, $x=$ "proportion of retired people", essentially measures the extent to which the values $x_{i}$ and $x_{j}$ of two regions $i$ and $j$ tend to be closer whenever $i$ and $j$ are neighbours - neighbours in the sense defined by a general spatial weights or spatial link matrix $V$, be it specified by the row-standardised spatial weights matrix $W$, the proximity matrix $G$ or the exchange matrix $E$. Here $x$ is a monadic tensor (vector) and $V$ is a dyadic tensor (matrix).

Flows $T=\left(t_{i j}\right)$ are themselves dyadic. The study of their spatial autocorrelation, investigating to which extent $t_{i j}$ and $t_{k l}$ are closer if $i j$ and $k l$ are "neighbours", seems to require a tetradic spatial link (spatial weights) $V=\left(v_{i j, k l}\right)$. For instance, one can set $v_{i j, k l}=F\left(d_{i k}, d_{j l}\right)$ where $F$ is a decreasing function of each of its two arguments, namely the distances $d_{i k}$ between distinct origins, and the distances $d_{j l}$ between distinct destinations (e.g. Black 1992 ; Bolduc et. al. 1995). For a given fixed origin $i=k$, one gets a functional relation of the form $v_{i j, i l}=F\left(d_{j l}\right)$ (common-origin link). Similarly, $v_{i j, k j}=F\left(d_{i k}\right)$ (common-destination link).

Tetradic spatial links seem formally necessary and intuitively appealing as far as the spatial autocorrelation of dyadic tensors (here the flows) is investigated: as a matter of fact, most contributions to flow autocorrelation refer in some way or another to the tetradic paradigm, as e.g. in Brandsma and Ketellapper (1979), Black and Thomas (1998), Berglund and Karlström (1999), Tiefelsdorf and Braun (1999), Fischer and Griffith (2008), LeSage and Pace (2008), LeSage and Polasek (2008), Polasek and Sellner (2010), or Behrens at al. (2012).

Tetradic tensors describe $n^{4}$ components, dyadic tensors (matrices) only $n^{2}$. Kronecker products of dyadic tensors yield tetradic block tensors of lower complexity. Such are the "origin-based", "destination-based" and "origin-todestination" row-standardized spatial weights ${ }^{o} W=I \otimes W,{ }^{d} W=W \otimes I$ and ${ }^{o d} W=W \otimes W$ (e.g. LeSage and Pace 2008; Fischer and Griffith 2008; and references therein). Allowing for distinct origin and destination weights $f \neq \rho$, the corresponding tetradic exchange matrices read as

$$
{ }^{o} \varepsilon_{i j, k l}=e_{i k} \rho_{j} \delta_{j l} \quad{ }^{d} \varepsilon_{i j, k l}=f_{i} \delta_{i k} \dot{e}_{j l} \quad{ }^{o d} \varepsilon_{i j, k l}=e_{i k} \dot{e}_{j l}
$$

where $\dot{E}(\rho, G, t)$ is the exchange matrix between destinations, weight compatible with $\dot{e}_{j \bullet}=\rho_{j}$. All proposals (9) have margins $\pi_{i j}:=\varepsilon_{i j, \bullet \bullet}=f_{i} \rho_{j}$, making global tetradic inertia $\hat{\Delta}$ identical to dyadic inertia $\Delta$ (see theorem below). To prove the assertion, consider $n$ origins indexed by $i, k, \ldots$ and $p$ destinations indexed by $j, l, \ldots$. The adjusted flow matrix $T=\left(t_{i j}\right)$ induces origin weights $f_{i}$, destination weights $\rho_{j}$ and independence quotients $q_{i j}$ as

$$
f_{i}:=\frac{t_{i \bullet}}{t_{\bullet \bullet}} \quad \rho_{j}:=\frac{t_{\bullet j}}{t_{\bullet \bullet}} \quad q_{i j}:=\frac{t_{i j}}{t_{i j}^{\text {theo }}}=\frac{t_{i j} t_{\bullet \bullet}}{t_{i \bullet} t_{\bullet j}}
$$


The non-negative, symmetric and normalized $n p \times n p$ tetradic exchange matrix $\varepsilon=\left(\varepsilon_{i j, k l}\right)$ defines a marginal dyadic weight $\pi_{i j}=\varepsilon_{i j, \bullet \bullet}$, as well as an autocorrelation index $\hat{\delta}(y)$ for a dyadic quantity $y=\left(y_{i j}\right)$ as (cf. equation (1)):

$$
\hat{\delta}(y)=\frac{\hat{\Delta}-\hat{\Delta}_{\mathrm{loc}}}{\hat{\Delta}} \quad \hat{\Delta}=\frac{1}{2} \sum_{i j k l} \pi_{i j} \pi_{k l}\left(y_{i j}-y_{k l}\right)^{2} \quad \hat{\Delta}_{\mathrm{loc}}=\frac{1}{2} \sum_{i j k l} \varepsilon_{i j, k l}\left(y_{i j}-y_{k l}\right)^{2} .
$$

Recall that $y$ must be a density, that is transforming under regional aggregation as an average. Quotients $y_{i j}=q_{i j}$ in (10) precisely behave in such a way, hence the following:

Theorem (on the dyadic approach as a particular case of the tetradic one): Define the $n p \times n p$ tetradic destination-based exchange matrix as $\varepsilon=E \otimes R$, where $E=\left(e_{i k}\right)$ is the $n \times n$ exchange matrix with margin $f$ in (10), and $R=\operatorname{diag}(\rho)$ is the $p \times p$ diagonal matrix with diagonal (and margin) $\rho$ in (10). Then relative autocorrelations coincide: $\hat{\delta}(q)=\delta$.

Proof: by definition of the Kronecker product $\otimes$ and the above, $\varepsilon_{i j, k l}=$ $e_{i k} \rho_{j} \delta_{j l}=\varepsilon_{k l, i j}$, with marginal dyadic weight $\pi_{i j}=\varepsilon_{i j, \bullet \bullet}=f_{i} \rho_{j}$. Then

$$
\begin{array}{r}
\hat{\Delta}_{\mathrm{loc}}=\frac{1}{2} \sum_{i j, k l} \epsilon_{i j, k l}\left(q_{i j}-q_{k l}\right)^{2}=\frac{1}{2} \sum_{i j, k l} e_{i k} \rho_{j} \delta_{j l}\left(q_{i j}-q_{k l}\right)^{2}= \\
=\frac{1}{2} \sum_{i j k} e_{i k} \rho_{j}\left(q_{i j}-q_{k j}\right)^{2}=\frac{1}{2} \sum_{i j} e_{i j} \sum_{k} \rho_{k}\left(q_{i k}-q_{j k}\right)^{2}=\frac{1}{2} \sum_{i j} e_{i j} D_{i j}^{\chi}=\Delta_{\mathrm{loc}} \\
\hat{\Delta}=\frac{1}{2} \sum_{i j, k l} f_{i} \rho_{j} f_{k} \rho_{l}\left(q_{i j}-q_{k l}\right)^{2}=\frac{1}{2} \sum_{i k} f_{i} f_{k} \sum_{j} \rho_{j}\left(q_{i j}-q_{k j}\right)^{2}=\Delta
\end{array}
$$

\section{Discussion and conclusions}

The exposed method constitutes a broadly applicable method for investigating flow autocorrelation, following the standard framework of spatial autocorrelation of regional features, in the weighted, multivariate case. Its implementation relies upon two matrices only, namely (i) the flow matrix itself $T$ of $T^{\prime}$, after diagonal adjustment, with relative margins $f$ and $\rho$, together with (ii) a proximity matrix $G$ (such as an adjacency matrix, weighted or not, or specifying the length of the boundary shared by regional pairs, etc.) roughly defining the inter-regional spatial structure. Interestingly enough, the procedure depends upon off-diagonal components of $T$ and $G$ only. In addition, the scheme contains a freely adjustable parameter $t$, controlling the decay of the distance deterrence and the resolution scale. 
Initially motivated by the wish to cover the question of flow autocorrelation in the usual framework, namely the dyadic formalism expressing Moran's $I$ or its multivariate generalization $\delta$, this investigation has first identified the chi2 dissimilarity between outflows or inflows as the natural dyadic measure of flow variability. The necessity to deal properly with the potentially troublesome status of stayer flows has led to a general algorithm of diagonal adjustment, of interest in itself and potentially meaningful in related issues, such as the MDS reconstruction of weighted oriented networks or the gravity modelling and fitting of flows. Decomposing the relative inertia $\delta$ as a weighted average of local indicators of spatial autocorrelation $\delta_{i}$ enables to map and to interpret the local patterns in terms of housing and job centres, with possible applications to regional clustering, or transportation planning. Finally, the demonstrated equivalence, under suitable conditions, between the tetradic and dyadic approaches, permits to embed the latter into the mainstream spatial autocorrelation paradigm, and should directly help guiding the construction and testing of autoregressive spatial econometrical models for flows.

Flow autocorrelation turns out to be highly significant for both case studies. Yet, local indicators of spatial autocorrelation are smaller for migration flows in Western US than for journey-to-work flows in Western Switzerland - an expected effect, considering that journey-to-work flows are spatially limited by the time budget available for commuting, while constraints related to economic environment or education are more important for migration.

Spatial distribution of residence and job locations, either attained daily (commuters) or following a further residence move (migrants), are arguably close. This could provide a rationale for the observed proximity between outand in-flows local autocorrelation exhibited in figures 1, 2, 3 and 4, as well as their particular relation to important places, either from a residential or job perspective, precisely.

\section{References}

1. Anselin, L. (1995). "Local indicators of spatial association - LISA", Geographical Analysis 27 , pp. $93-115$

2. Bavaud, F. (1998). "Models for Spatial Weights: A Systematic Look", Geographical Analysis 30 , pp. $153-171$

3. Bavaud, F. (2013). "Testing spatial autocorrelation in weighted networks: the modes permutation test", Journal of Geographical Systems 15, pp. 233-247

4. Bavaud, F. (2014). "Spatial weights: constructing weight-compatible exchange matrices from proximity matrices". In: Duckham, M. et al. (Eds.): GIScience 2014, LNCS 8728, pp. 81-96. Springer

5. Behrens, K., Ertur, C., and Koch, W. (2012). "Dual gravity: Using spatial econometrics to control for multilateral resistance", Journal of Applied Econometrics 27, pp. 773-794

6. Berger, J. and Snell, J.L. (1957). "On the concept of equal exchange", Behavioral Science 2, pp. $111-118$

7. Berglund S. and Karlström, A. (1999). "Identifying local spatial association in flow data", Journal of Geographical Systems 1, pp. 219-236

8. Bishop, Y. M., Fienberg, S. E., and Holland, P.W. (1975). "Discrete Multivariate Analysis: Theory and Practice". The MIT Press, Cambridge, Massachusetts 
9. Bivand, R. S., Pebesma, E. J., Gómez-Rubio, V., and Pebesma, E. J. (2008). "Applied spatial data analysis with R". New York: Springer

10. Black, W. R. (1992). "Network autocorrelation in transportation network and flow systems", Geographical Analysis, 24, pp. 207-222

11. Black, W. R. and Thomas, I. (1998). "Accidents on Belgiums motorways: A network autocorrelation analysis", Journal of Transport Geography, 6, 23-31

12. Bolduc D., Laferriere R. and Santarossa G. (1995). "Spatial autoregressive error components in travel flow models: An application to aggregate mode choice". In New Directions in Spatial Econometrics, Anselin L., Florax R.J. (eds). Springer, Berlin, Heidelberg, New York, pp. $96-108$

13. Brandsma A.S. and Ketellapper R.H. (1979). "A biparametric approach to spatial autocorrelation", Environment and Planning A 11, pp. 51-58.

14. Chung, F.R.K. (1997). "Spectral Graph Theory". AMS

15. Cover, T. and Thomas, J. (1991). "Elements of Information Theory". Wiley

16. Cliff, A. D. and Ord, J. K. (1981). "Spatial processes". Pion

17. Fischer, M. M. and Griffith, D. A. (2008). "Modeling spatial autocorrelation in spatial interaction data: an application to patent citation in the European Union", Journal of Regional Science, 48(5), 969-989.

18. Hillberry, R. and Hummels, D. (2008). "Trade responses to geographic frictions: A decomposition using micro-data". European Economic Review, 52, pp. 527-550

19. Kondor, R. I. and Lafferty, J. (2002). "Diffusion kernels on graphs and other discrete input spaces". In ICML (Vol. 2), pp. 315-322

20. LeSage J. and Pace R.K. (2008). "Spatial econometric modeling of origin-destination flows", Journal of Regional Science 48, pp. 941-967

21. LeSage J. and Polasek W. (2008). "Incorporating Transportation Network Structure in Spatial Econometric Models of Commodity Flows", Spatial Economic Analysis 3, pp. $225-245$

22. Llano-Verduras, C., Minondo, A., and RequenaSilvente, F. (2011). "Is the border effect an artefact of geographical aggregation?". The World Economy, 34, pp. 1771-1787

23. Polasek, W. and Sellner, R. (2010). "Spatial Chow-Lin methods for data completion in econometric flow models" (No. 255). Reihe Ökonomie/Economics Series, Institut für Höhere Studien (IHS).

24. Tiefelsdorf M. and Braun, G. (1999). "Network Autocorrelation in Poisson Regression Residuals: Inter-district Migration Patterns and Trends within Berlin", paper presented at the 11th European Colloquium on Quantitative and Theoretical Geography, Durham City, England, September 3-7

\section{Appendix: computational details of diagonal flow adjustment}

Define a family of independent flows by the model distribution $p_{i j}^{\text {theo }}=\beta_{i} \gamma_{j}$ where $\beta$ and $\gamma$ are unknown distributions. The free parameters are estimated so as the relative complete flows $p$, of the form (7) $p_{i j}=\sigma_{i} \delta_{i j}+\mu a_{i j}$, is close as possible from independent relative flows $p^{\text {theo }}$, in the sense that the Kullback-Leibler divergence or relative entropy

$$
K\left(p \| p^{\text {theo }}\right)=\sum_{i j} p_{i j} \ln \frac{p_{i j}}{\beta_{i} \gamma_{j}}=\sum_{i} \sigma_{i} \ln \frac{\sigma_{i}}{\beta_{i} \gamma_{i}}+\sum_{i j} \mu a_{i j} \ln \frac{\mu a_{i j}}{\beta_{i} \gamma_{j}}
$$

must be minimum, under the constraints

$$
\mu=1-\sigma_{\bullet} \geq 0 \quad \sigma_{i}, \beta_{i}, \gamma_{j} \geq 0 \quad \sum_{i} \beta_{i}=\sum_{j} \gamma_{j}=1 .
$$


Setting to 0 the derivative by $\sigma_{i}$ yields with $\mu=1-\sigma_{\bullet}$ :

$$
\ln \frac{\sigma_{i}}{\beta_{i} \gamma_{i}}+1-\sum_{i j} a_{i j} \ln \frac{\mu a_{i j}}{\beta_{i} \gamma_{j}}-\sum_{i j} a_{i j}=\ln \frac{\sigma_{i}}{\beta_{i} \gamma_{i}}-\ln \mu-K(a \| \beta \gamma)=0
$$

where $K(a \| \beta \gamma):=\sum_{i j} a_{i j} \ln \frac{a_{i j}}{\beta_{i} \gamma_{j}}$ is the "movers" relative entropy, which, incidentally, also appears in the likelihood ratio of the so-called quasi-independence models (e.g. Bishop et al. 1975). Derivating w.r.t. $\beta_{i}$ under the constraint $\sum_{i} \beta_{i}=1$ (and multiplier $\lambda$ ) yields

$$
-\frac{\sigma_{i}}{\beta_{i}}-\mu \frac{a_{i} \bullet}{\beta_{i}}=-\lambda \quad \beta_{i} \lambda=\sigma_{i}+\mu a_{i} \bullet \quad \Rightarrow \quad \lambda=1
$$

in view of $\sigma_{\bullet}+\mu=1$. Hence $\beta_{i}=\sigma_{i}+\mu a_{i \bullet}$. Similarily, $\gamma_{j}=\sigma_{j}+\mu a_{\bullet j}$. Finally,

$\sigma_{i}=\mu \beta_{i} \gamma_{i} \exp K(a \| \beta \gamma) \quad \mu=1-\sigma_{\bullet} \in(0,1) \quad \beta_{i}=\sigma_{i}+\mu a_{i} \bullet \quad \gamma_{j}=\sigma_{j}+\mu a_{\bullet j}$

where $a_{i j}=m_{i j} / m_{\bullet \bullet}$ is the known proportion of movers from $i$ to $j$ (section 2.4), from which the unknown $\sigma, \mu, \beta, \gamma$ are to be estimated.

Equations (11) can be iteratively solved from some initial solution such as $\beta_{i}^{0}=a_{i \bullet}, \gamma_{j}^{0}=a_{\bullet j}$ and $\sigma_{i}^{0}=a_{i \bullet} a_{\bullet i}$ until convergence, which occurs provided $a_{i \bullet}>0$ and $a_{\bullet j}>0$ for all origins $i$ and destinations $j$. If needed, the latter conditions can be insured by considering augmented flows $t_{i j}+1$ instead of $t_{i j}$, a choice adopted for convenience in the case studies of section 3 , and justifiable in a Bayesian framework (Laplace rule of succession).

More generally, $a_{i \bullet}+a_{\bullet}=0$ makes $i$ irrelevant (a "non-region"). $a_{i \bullet}=0$ and $a_{\bullet i}>0$ makes $\beta_{i}=\sigma_{i}=0$ (absorbing destination). Similarly, $a_{i}>0$ and $a_{\bullet i}=0$ makes $\gamma_{i}=\sigma_{i}=0$ (transient origin). Finally, $a_{i \bullet}+a_{\bullet i}>0$ and $a_{i} a_{\bullet}=0$ for all $i$ splits the regions between two non-intersecting sets, namely transient origins and absorbing destinations, with $\sigma_{\bullet}=0$ and $\mu=1$, characterizing incommensurate, "rectangular" flows. 